\title{
AXIOMATIZATION OF DIVISOR METHODS AND WEAKLY DEGRESSIVE PROPORTIONALITY
}

\section{Piotr Dniestrzański}

\begin{abstract}
The paper analyzes the differences between weakly degressive proportionality and proportional allocations represented by divisor methods. The analysis is accomplished by the axiomatization of divisor methods in line with Balinski and Young.
\end{abstract}

Keywords: degressive proportionality, fair division, axiomatization of divisor methods.

JEL Classification: D63, D69

DOI: $10.15611 / \mathrm{dm} .2017 .14 .02$

\section{Introduction}

In this paper we will focus on the presentation of free distributions of divisor methods which have a strong position in the proportional apportionment problem. They are the only methods that satisfy natural axioms and avoid the so-called paradoxes, i.e. unexpected and illogical properties. In some practical cases associated with the division of goods subject to the consent of all participants, proportional allocations give way to apportionment, which is closer to equal proportions. This happens, for example, when the values of the agents participating in the division are at wide variance, and proportional allocations could result in disregarding the smallest agents. To avoid such a case, the greater agents are allocated a smaller amount of a good than their proportional share. This bias is in favor of smaller agents, because they receive more goods than their proportional amount. The resulting allocations are closer to an equal division if they satisfy a further obvious condition stipulating that a greater agent may not get less than a smaller agent. Allocations, whose ratios of the good's amount and the agent's value decrease (do not increase) as the agent's value increases, also satisfying the so-called weak monotonicity, are said to be degressively proportional (or strictly degressively proportional). In cases of indivisible goods, the so-called weakly

Piotr Dniestrzański

Wrocław University of Economics

email: piotr.dniestrzanski@ue.wroc.pl

ORCID: 0000-0001-6131-8635 
degressive proportionality (known also as unrounded degressive proportionality - UDP) is also considered, whose decreasing ratio of the good's number to entitlement can be distorted as a result of rounding.

In this paper we analyze the differences between weakly degressive proportionality and proportional allocations at the level of axioms. Using the classic theorem of Balinski and Young [1982] who present the full axiomatization of divisor methods, we investigate which of the five axioms are also satisfied in cases of a weakly degressive proportionality.

\section{Axioms of divisor methods}

Divisor methods are widely applied in the apportionment problem of indivisible goods, in particular regarding the allocation of seats in legislative bodies. Let $\mathrm{H}$ be the number of a good to be distributed among $\mathrm{n}$ agents with entitlements $p_{1}, p_{2}, \ldots, p_{n}\left(p_{i}>0\right)$ and let $V$ be the sum of entitlements (values) of these agents $V=p_{1}+p_{2}+\ldots+p_{n}$. A divisor method is when the number of a good allocated to the agent with entitlement $p_{i}$ equals $s_{i}=\left[\frac{p_{i}}{d}\right]$, where the value $d$, called divisor, is chosen so as to satisfy the equation

$$
\left[\frac{p_{1}}{d}\right]+\left[\frac{p_{2}}{d}\right]+\ldots+\left[\frac{p_{n}}{d}\right]=H
$$

and $[x]$ denotes rounding of the number $\mathrm{x}$ to integer. Rounding can be upwards, downwards, to the nearest integer or with moving thresholds. A complete theory of divisor methods, with reference to degressive proportionality, is given among others by Pukelsheim [2014]. Young [1995] analyzes the properties of divisor methods against the background of fair apportionment. Karpov [2008] presents the results of research into the exactness of the various methods of proportional allocation in electoral systems.

Example 1. Let $H=20$ be the number of a good to be allocated, entitlements are given by a vector $p=(1,2,5)$, and $s_{i}=\left[\frac{p_{i}}{d}\right]$, with upwards rounding. The allocation of the good $H$ is then given by the vector $s=\left(s_{1}, s_{2}, s_{3}\right)=$ $(3,5,12)$. One of the divisors satisfying the equation (1) is $d=9$. The respective agents are allocated the following numbers of the good: $s_{1}=3, s_{2}=5$, 
$S_{3}=12$. Given function A from the set $\bigcup_{n=1}^{\infty}\left(R_{+}^{n} \times R_{+}\right)$, where $R_{+}^{n}$ is the set of all entitlement vectors, and $R_{+}$is the set of all numbers of the good, to the set $\bigcup_{n=1}^{\infty}\left(R_{+}^{n}\right)$, where $R_{+}^{n}$ denotes the set of all allocation vectors of the good, is said to be the allocation function.

The vector $s=A(p, H) \in \bigcup_{n=1}^{\infty}\left(R_{+}^{n}\right)$ is called the apportionment, where $s_{i}=A(p, H)\left(p_{i}\right)$ is the number of the good allocated in this solution to the agent with the entitlement $p_{i}$. It is said that the agent $p_{i}$ gets the good $s_{i}$ under the apportionment $A$. In all our analyzes and examples we deal with indivisible goods, therefore the considered apportionments are integer. At times, the allocation function $\mathrm{A}$ is called just allocation for the sake of convenience. In the problem of proportional apportionments there are many expectations (axioms, conditions) imposed on allocation functions. We highlight five of them that are key in further considerations.

Unbiasedness. The allocation $A$ is unbiased, if $A(\pi(p), H=\pi(p, H))$ for every permutation $\pi$ of $n$ elements. Unbiasedness implies that the number of the good allocated to each agent is determined exclusively by the value of agent's entitlement, in other words the apportionment is independent of the ordering of agents.

Pairwise consistency. The allocation A is said to be pairwise consistent, if ith and jth coordinates of vectors $A(p, H)$ and $A\left(\left(p_{i}, p_{j}\right), s_{i}+s_{j}\right)$, where $s_{i}=A(p, H)\left(p_{i}\right)$ and $s_{j}=A(p, H)\left(p_{j}\right)$, are equal. Pairwise consistency means that if agents with entitlements $p_{i}$ and $p_{j}$, as a result of the allocation $A$, get $s_{i}$ and $s_{j}$ of the good, respectively, therefore in cases when they are the only agents participating in the apportionment, and the total number of the allocated good equals exactly $s_{i}+s_{j}$, they would have also obtained the good $s_{i}$ and $s_{j}$.

Homogeneity. The allocation $A$ is said to be homogenous, if $A(\lambda p, H)=$ $A(p, H)$ for every integer $\lambda$. Homogeneity therefore implies that a proportional increase of entitlements of all agents, at the integer scaling, does not change the result of apportionment. 
Exactness. The allocation $A$ is said to be exact, if $A(p, H)=p \cdot(H / V)$ holds always when all coordinates of the vector $p \cdot(H / V)$ are integer. Exactness indicates that if a strictly proportional apportionment is possible, it has to be a result of the allocation.

Weak monotonicity. The allocation $A$ is said to be weakly monotone, if the inequality $p_{i}>p_{j}$ implies the inequality $s_{i} \geq s_{j}$. Weak monotonicity indicates that no agent with greater entitlement may be allocated fewer goods than an agent with a smaller entitlement.

Divisor methods play a significant role in the problem of proportional apportionments. They avoid the so-called paradoxes, whereas largest remainder methods allow the paradoxes. The remarkableness of the divisor methods is demonstrated by the following theorem.

Theorem [Balinski, Young 1982]. The allocation is unbiased, pairwise consistent, homogenous, exact and weakly monotone if and only if it is a divisor method.

Among all divisor methods, Webster's method with rounding to the nearest integer, is especially worth considering. To justify its remarkableness let us introduce the following concept. The standard apportionment among two agents is called the apportionment with good $\mathrm{H}$ distributed according to their entitlements $p_{1}$ and $p_{2}$ in quantities $s_{1}=\left[q_{1}\right]$ and $s_{2}=\left[q_{2}\right]$, where $q_{1}=\frac{H p_{1}}{p_{1}+p_{2}}$ and $q_{2}=\frac{H p_{2}}{p_{1}+p_{2}}$, and the rounding is to the nearest integer. If fractional parts $q_{1}$ and $q_{2}$ are exactly equal to 0.5 , then the Webster apportionment is both $s_{1}=\left[q_{1}-0.5\right], s_{2}=\left[q_{2}+0.5\right]$, as well as $s_{1}=\left[q_{1}+0.5\right], s_{2}=\left[q_{2}-0.5\right]$.

Balinski and Young [1982] proved that Webster's method is the only method which is pairwise consistent with the standard apportionment for two agents.

\section{Weakly (unrounded) degressive proportionality}

Unrounded degressive proportionality (UDP) is the middle solution between equity and proportionality. Large agents get less as a result of the UDP allocation, whereas small agents get more. The extreme cases of the UDP are 
equal apportionment and proportional apportionment. Let us consider the allocation $A_{f}$ of the form

$$
A_{f}(p, H)=\left(\frac{\left[f\left(p_{1}\right)\right]}{d}, \frac{\left[f\left(p_{2}\right)\right]}{d}, \ldots, \frac{\left[f\left(p_{n}\right)\right]}{d}\right),
$$

where $f: R_{+} \rightarrow R_{+}$is a given function. The allocation $A_{f}$ is said to satisfy the unrounded degressive proportionality (UDP), if the following conditions are satisfied

$$
\begin{gathered}
p_{i}>p_{j} \text { implies } q_{i} \geq q_{j} \\
p_{i}>p_{j} \text { implies } \frac{q_{i}}{p_{i}} \leq \frac{q_{j}}{p_{j}} \\
\sum_{i=1}^{n} s_{i}=H,
\end{gathered}
$$

where

$$
q_{i}=\frac{f\left(p_{i}\right)}{d}, \quad q_{j}=\frac{f\left(p_{j}\right)}{d} .
$$

The apportionment resulting from the function $A_{f}$ will be called the UDP apportionment. As can be easily shown [Dniestrzański 2014b], given the differentiable function $f$, the allocation $A_{f}$ is UDP if and only if $\frac{d f(x)}{d x} \in\left\langle 0, \frac{f(x)}{x}\right\rangle$. If $\frac{d f(x)}{d x} \equiv 0$, we obtain the equal apportionment, and if $\frac{d f(x)}{d x}=\frac{f(x)}{x}$ we obtain the proportional apportionment.

Example 2. Following Example 1, let: $\mathrm{H}=20, p=(1,2,5)$, rounding up and $f(x)=\sqrt{x}$. Given the allocation function $A_{f}$, we obtain the apportionment $A_{f}(p, H)=A_{f}((1,2,5), 20)=(5,6,9)$.

Examples 1 and 2 (Table 1) yielded significantly different results. The differences are mostly remarkable in the case of the smallest and the largest agent, as a consequence of adjusting the entitlements, that actually happens due to the function $f(x)=\sqrt{x}$. 
Table 1. Comparison of apportionments delivered in Examples 1 and 2

\begin{tabular}{|l|c|c|c|c|}
\hline Values of agents & $p_{1}=1$ & $p_{2}=2$ & $p_{3}=5$ & \\
\hline Webster's allocation (example 1) & $s_{1}=3$ & $s_{2}=5$ & $s_{3}=12$ & $H=20$ \\
\hline UDP allocation $f(x)=\sqrt{x}$ (example 2) & $s_{1}=5$ & $s_{2}=6$ & $s_{3}=9$ & $H=20$ \\
\hline
\end{tabular}

Source: own elaboration.

We shall now analyze whether the five properties described in Section 2, yielding a full axiomatization of divisor methods, are satisfied in cases of UDP allocations, i.e. those satisfying the conditions (2)-(5).

Unbiasedness - this condition is obviously satisfied.

Pairwise consistency. This condition is satisfied by allocation $A_{f}$, given any function $f$, even the conditions (3) and (4) do not have to be satisfied. This is a consequence of the fact that UDP with function $f$ is equivalent to a divisor method after transforming the vector of entitlements $p$ into the vector of entitlements $p_{f}=\left(f\left(p_{1}\right), f\left(p_{2}\right), \ldots, f\left(p_{n}\right)\right)$.

Homogeneity - this condition is obviously satisfied.

Exactness. This condition is not satisfied. For example, given $f=$ const, $\mathrm{H}=20, p=(1,2,3)$ and upwards rounding, we obtain $A_{f}((1,2), 6)=(3,3) \neq$ $(1,2) \cdot 2$.

Weak monotonicity. This condition is satisfied by definition of the UDP - the equation (4) must hold.

All five axioms characterizing divisor methods are very natural, and the requirement that proportional allocations should satisfy them seems obvious. It turns out that as many as four are satisfied also in the case of weakly degressive proportionality. The only axiom that is not indispensable for the UDP, and even undesirable, is consistency. The failure to meet this axiom is not unusual because it always compels the strictly proportional apportionment whenever possible. However, knowing that the remaining four axioms are satisfied, one may attempt to look for one or more axioms which will characterize the weakly degressive proportionality along with the current four. This in turn could enable a more in-depth analysis of the UDP. 
In practical applications, the so-called boundary conditions are imposed on weakly degressive proportional allocations (as well as on proportional allocations). They are typically in the form of specifying the minimum and maximum number of the good that can be allocated to agents. For example, in the case of the European Parliament (in compliance with the Treaty of Lisbon, the composition of the European Parliament must be degressively proportional), the numbers of seats apportioned to the member states must belong to the set $\{6,7, \ldots, 96\}$. The boundary conditions can distort the original idea as much as possible. However, if they are "reasonably" associated with the remaining components of allocations (the size of the good to be distributed, the number and entitlements of the agents), one may also in this case analyze the allocation problem from the viewpoint of axioms. The analyses of boundary conditions influencing degressively proportional allocations are for example in papers by Łyko [2013], and Dniestrzański, Łyko [2014]. Dniestrzański [2014a] puts forward an approach to investigate the level of degression in a given allocation (or a method).

\section{Conclusion}

Degressive proportionality represents a key element of the theory of fair distribution. It is an alternative to proportional allocation, especially when entitlements of agents vary significantly. In contrast with proportional and equal allocations, most often there are a lot of solutions satisfying the conditions of degressive proportionality. In practical applications, the selection of one solution can be a serious problem. This is exemplified by the case of the European Parliament, where despite legally binding stipulations consecutive parliamentary terms have failed to reach the apportionment of the seats among the member states that fully satisfies the idea of degressive proportionality. The analysis of this allocation method at the level of axioms may contribute to its better understanding, and as a consequence, to enable its implementation. This paper investigated the differences between degressive proportionality (in its weak variant, as the UDP) and the divisor methods underlying proportionate allocations. 


\section{Bibliography}

Balinski M., Young H.P. (1982). Fair Representation: Meeting the Ideal of One Man, One Vote. Yale University Press.

Dniestrzański P. (2014a). Proposal for measure of degressive proportionality, Procedia Social and Behavioral Sciences. No 110. Pp. 140-147.

Dniestrzański P. (2014b). Kompromis z Cambridge - pomiędzy równościa a proporcjonalnościa [Cambridge compromise - between equity and proportionality]. Studia Ekonomiczne. Badania ekonometryczno-statystyczne w teorii i praktyce. Wydawnictwo Uniwersytetu Ekonomicznego w Katowicach. Pp. 50-60.

Dniestrzański P., Łyko J. (2014). Influence of boundary conditions of digressively proportional division on the potential application of proportional rules. Procedia - Social and Behavioral Sciences. No 109. Pp. 722-729.

Karpov A. (2008). Measurement of disproportionality in proportional representation systems. Mathematical and Computer Modelling. No 48. Pp. 1421-1438.

Łyko J. (2013). Boundary, internal and global measure of the degression of distribution. Procedia - Economics and Finance. No 5. Pp. 519-523

Pukelsheim F. (2014). Proportional Representation. Apportionment Methods and Their Applications. Springer

Young H.P. (1995). Equity: In Theory and Practice. Princeton University Press. 